\title{
Acupuncture Treatment for Chronic Pelvic Pain in Women: A Systematic Review and Meta-Analysis of Randomized Controlled Trials
}

\author{
Soo-Hyun Sung $\mathbb{D}^{1},{ }^{1}$ Angela-Dong-Min Sung $\mathbb{D}^{2},{ }^{2}$ Hyun-Kyung Sung, ${ }^{3}$ Tteul-E-Bom An, \\ Kyeong Han Kim, ${ }^{5}$ and Jang-Kyung Park $\mathbb{D}^{6}$ \\ ${ }^{1}$ Department of Pathology, College of Korean Medicine, Dae-gu Haany University, Daegu 38610, Republic of Korea \\ ${ }^{2}$ Department of Preventive Medicine, College of Korean Medicine, Sangji University, Wonju 26339, Republic of Korea \\ ${ }^{3}$ Department of Korean Pediatrics, College of Korean Medicine, Semyung University, Jechon 27136, Republic of Korea \\ ${ }^{4}$ Department of Obstetrics and Gynecology, College of Korean Medicine, Dae-gu Haany University, Daegu 38610, Republic of Korea \\ ${ }^{5}$ Department of Preventive Medicine, College of Korean Medicine, Woosuk University, Wanju 55338, Republic of Korea \\ ${ }^{6}$ Department of Obstetrics and Gynecology, College of Korean Medicine, Sangji University, Wonju 26339, Republic of Korea
}

Correspondence should be addressed to Jang-Kyung Park; vivat314@naver.com

Received 7 June 2018; Accepted 12 September 2018; Published 27 September 2018

Guest Editor: Kyung-Hwa Jung

Copyright (C) 2018 Soo-Hyun Sung et al. This is an open access article distributed under the Creative Commons Attribution License, which permits unrestricted use, distribution, and reproduction in any medium, provided the original work is properly cited.

\begin{abstract}
Aim of the Study. This systematic review and meta-analysis aims to evaluate the current evidence from randomized controlled trials (RCTs) related to the effectiveness and safety of acupuncture treatment (AT), including electroacupuncture or threadembedding therapy in combination with modern technology, for chronic pelvic pain (CPP) in women. Materials and Methods. We searched 12 electronic databases up to December 2017. All randomized controlled trials evaluating the effect of AT for CPP were considered. Results. Four RCTs with 474 participants were included. The methodological quality of included studies was generally low. The results of meta-analysis of two studies showed that AT combined with conventional treatment (CT) was associated with significantly reduced CPP, based on the total effectiveness rate $(\mathrm{n}=277$, mean difference $=1.29$, confidence interval $=1.13$ to 1.47 , $\left.\mathrm{P}=0.0001, \mathrm{I}^{2}=0 \%\right)$. Conclusions. This review suggests the potential of AT combined with CT compared to CT alone for treating female CPP. However, there is insufficient evidence to conclude that AT can be recommended as a complementary and alternative (CAM) treatment for women with CPP. To draw a firm conclusion, future studies should require not only lager, more rigorously designed RCTs but also research on different AT types. Protocol Registration Number. This study is registered with PROSPERO 2018 (CRD42018088627).
\end{abstract}

\section{Introduction}

Chronic pelvic pain (CPP) is noncyclic pain of more than 6 months that localizes in the pelvis, the anterior abdominal wall at or below the umbilicus, the lumbosacral region of the spine, or the buttocks $[1,2]$. A total of $14.7 \%$ of women aged 18-50 years in the United States experience CPP within the prior three months [3]. Severe CPP not only causes functional disability in patients, but also reduces quality of life $[1,4]$.

Although there is no clear understanding of the mechanism of CPP, the European Association of Urology (EAU) guidelines suggest that inflammation or infection of somatic or visceral tissue, central nervous system (CNS) activity, and emotional, cognitive, behavioural, and sexual components are involved [5]. The guidelines include a description of the diagnosis and treatment of CPP according to a predefined mechanism [5].

In traditional Korean medicine, the main causes of CPP are thought to be static blood or depression of seven emotions and are treated with acupuncture or herbal medicine [6, 7].

Acupuncture has long been used and is effective in relieving pain and is also minimally invasive, inexpensive, and safe $[8,9]$. 
Acupuncture used in combination with modern technology, for example, electroacupuncture (EA), delivers electrical current through acupuncture, and acupoint threadembedding therapy (TET) maximizes stimulation by inserting thread into meridian points [10].

Recently, comprehensive guidelines for the diagnosis and treatment of CPP have been developed by the EAU [5]. However, there is no evidence-based complementary and alternative medicine (CAM) treatment. Moreover, no published systematic review has determined whether acupuncture treatment (AT) (e.g., classic acupuncture, EA, and TET) for CPP is safe and effective.

This systematic review and meta-analysis aims to evaluate current evidence from randomized controlled trials (RCTs) to assess the effectiveness and safety of AT for CPP.

\section{Methods}

2.1. Protocol and Registration. This systematic review was registered in the PROSPERO 2018 (available from http://www .crd.york.ac.uk/PROSPERO/display_record.php?ID=CRD42018088627).

2.2. Data Sources and Searches. The following electronic databases were searched to identify relevant studies for inclusion in the review from inception to December 2017: the Cochrane Central Register of Controlled Trials (CENTRAL), MEDLINE, EMBASE, CINAHL Plus, two Chinese databases (the China National Knowledge Infrastructure (CNKI) and Wanfang), and six Korean databases (the National Digital Science Library (NDSL), the Korean Traditional Knowledge Portal, KoreaMed, the Oriental Medicine Advanced Searching Integrated System (OASIS), the Research Information Sharing Service (RISS), and The National Library of Korea).

The search terms used were ("chronic pelvic pain" OR "chronic pelycalgia" OR "chronic pain of pelvic" OR "chronic pelvic ache") AND ("acupuncture" OR "acupoint” OR "needling” OR "electroacupuncture” OR “electroacupuncture" OR "electric acupuncture" OR "hand acupuncture" OR "scalp acupuncture" OR "auricular acupuncture" OR "ear acupuncture") AND ("Randomized controlled trial” OR “randomized clinical trial”).

2.3. Study Selection. All RCTs evaluating the effect of AT of CPP were included. Nonrandomized trials, animal or cell studies, and reviews were excluded. Women participants diagnosed with CPP were considered. Any type of AT (e.g., classic acupuncture, electroacupuncture, scalp acupuncture, auricular acupuncture, and thread-embedding therapy) for treating CPP was included. AT that does not involve the insertion of needles into the skin (e.g., acupoint pressure, or acupressure) was not considered. We included RCTs comparing AT with no treatment, placebo/sham treatment, or conventional treatment (CT). RCTs that assessed the combined effects of AT plus CT were also included when the identical CT was applied to both groups. Our primary outcome measure was the patient-reported pain score (e.g., visual analogue scale, numeric rating scale for CPP, or total effectiveness rate for $\mathrm{CPP}$ ). As secondary outcomes, we examined quality of life, activity score, and adverse events (AEs).

2.4. Data Extraction. Two of the authors (A. D. Sung and H. K. Sung) independently reviewed and screened the titles and abstracts of the retrieved studies based on the predefined eligibility criteria. Two independent reviewer (S. H. Sung and T. E. An) extracted the following data from the included studies: author information, sample size, types of diseases, intervention and control groups, outcome measures, main results, and any adverse events. Any disagreements arising between the reviewers during this process were resolved through discussion with a third author (J. K. Park).

2.5. Assessment of Risk of Bias (ROB). Two authors (S. H. Sung and K. H. Kim) independently evaluated the risk of bias of the included RCTs using the Cochrane Handbook V.5.1.0 [15]. This tool consists of seven domains, but we assessed the following six: random sequence generation, allocation concealment, blinding of participants and personnel, blinding of outcome assessments, incomplete outcome data, and selective reporting. For each domain, the risk of bias for each study was assessed according to three categories: low risk (L), high risk $(\mathrm{H})$, or unclear $(\mathrm{U})$. Disagreements encountered during the process were settled by a third author (J. K. Park) through discussion.

2.6. Data Analyses. For meta-analysis, we used RevMan software (Version 5.3.5 for windows; the Nordic Cochrane centre, Copenhagen, Denmark) [16]. Pooled dichotomous data were expressed as a risk ratio (RR) with $95 \%$ confidence interval (CI). In this case, we used a random-effects model for analysis and addressed heterogeneity among the included studies using the $\mathrm{I}^{2}$ test. $\mathrm{I}^{2}$ values above $50 \%$ or P values less than 0.10 showed considerable heterogeneity [11]. A summary of the findings was discussed in the results when a metaanalysis was not assessed.

\section{Results}

3.1. Study Selection and Description. The searches identified 117 potentially relevant studies, of which 4 RCTs (English databases: $n=1$; Chinese databases: $n=3$ ) met our inclusion criteria (Figure 1). Details of the included RCTs are summarized in Table 1. Three [12-14] of the 4 RCTs were conducted in China and published in Chinese. The remaining study [11] was conducted in Egypt and published in English.

3.2. Participants. A total of $474 \mathrm{CPP}$ patients were included in the review. The number of participants was 250 in the experimental group and 224 in the control group. Three of the included RCTs assessed clinical conditions: pelvic adhesion $[12]$ and pelvic inflammatory disease $[13,14]$.

3.3. Interventions. The types of AT in the RCTs varied: warm acupuncture with moxibustion on the handle of the needle was used in two studies [12, 13]; and EA [11] and TET combined with auricular acupuncture [14] were utilized in one study each. 


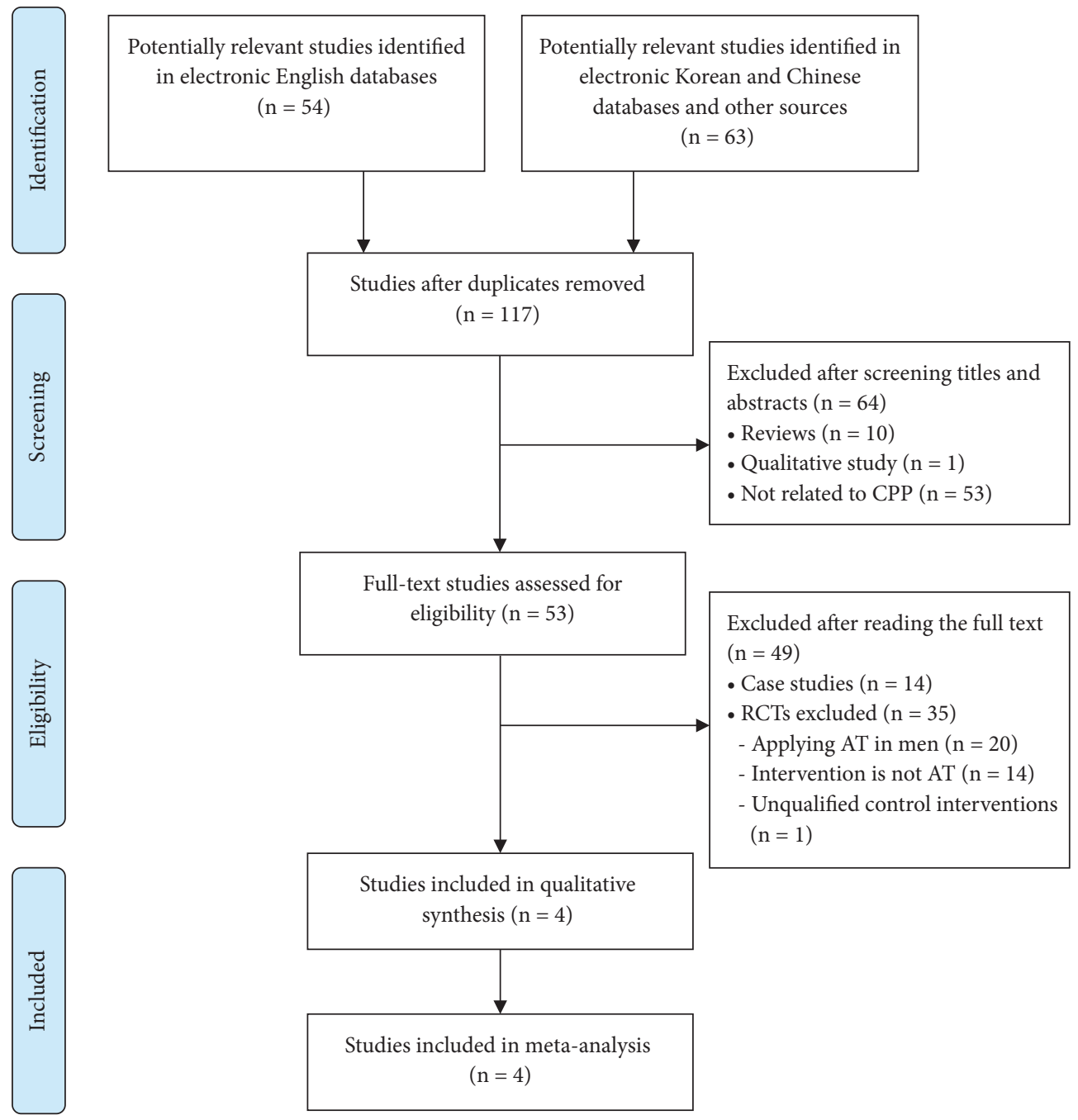

FIgURE 1: Flowchart of the RCT selection process. CPP: chronic pelvic pain; CCTs: controlled clinical trials; RCTs: randomized controlled trials; EAHM: external application of herbal medicine.

Two of the included RCTs compared AT, including EA and TET combined with auricular acupuncture, with CT $[11,14]$. Two other trials compared warm acupuncture plus CT with CT alone $[12,13]$.

\subsection{Outcomes}

3.4.1. Acupuncture Treatment versus Conventional Treatment. Acupuncture treatment was compared with conventional treatment in two RCTs [11, 14], of which one contrasted EA with inferior hypogastric plexus blockade [11], while another compared TET plus auricular acupuncture with levofloxacin administration [14].

Two meta-analyses [11, 14] that compared the primary outcome of the total effectiveness rate (TER) for CPP between AT and conventional treatment showed no significant difference between the groups [Figure 2(a), mean difference $(\mathrm{MD})=1.00$, confidence interval $(\mathrm{CI})=0.66$ to $1.53, \mathrm{P}$ $\left.=0.99, \mathrm{I}^{2}=92 \%\right]$. Amin [11] reported significant pain relief, measured with the visual analogue scale (VAS), in the AT group $(\mathrm{P}<0.001)$ and conventional treatment group $(\mathrm{P}<0.001)$.

3.4.2. Acupuncture Treatment Plus Conventional Treatment versus Conventional Treatment. For the primary outcome of TER for CPP, data extracted from two RCTs [12, 13] showed significantly superior improvement in the experimental group compared to the control group [Figure 2(b), $\mathrm{MD}=1.29, \mathrm{CI}=1.13$ to $\left.1.47, \mathrm{P}=0.0001, \mathrm{I}^{2}=0 \%\right] . \mathrm{Li}[12]$ reported significant efficacy based on the numeric rating scale (NRS) $(\mathrm{P}<0.05)$.

3.4.3. Adverse Events. One RCT [11] reported that AEs did not occur.

3.5. Cochrane Risk of Bias Assessment. The risk of bias of the included studies is presented in Figure 3. No included RCTs [8] mentioned the method of randomization or allocation concealment. Blinding of participants and practitioners was not performed in all of the included studies [8] due to 


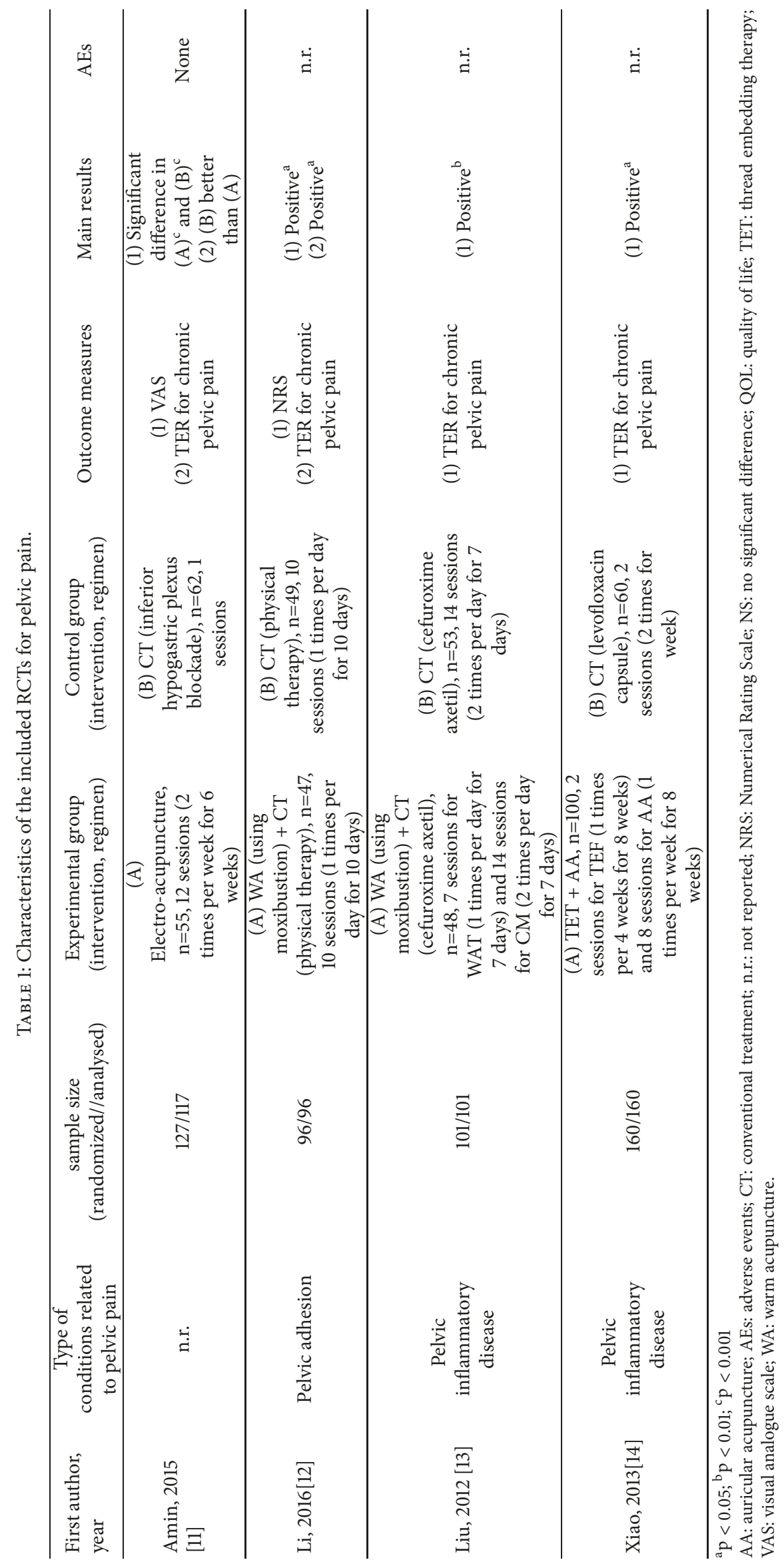




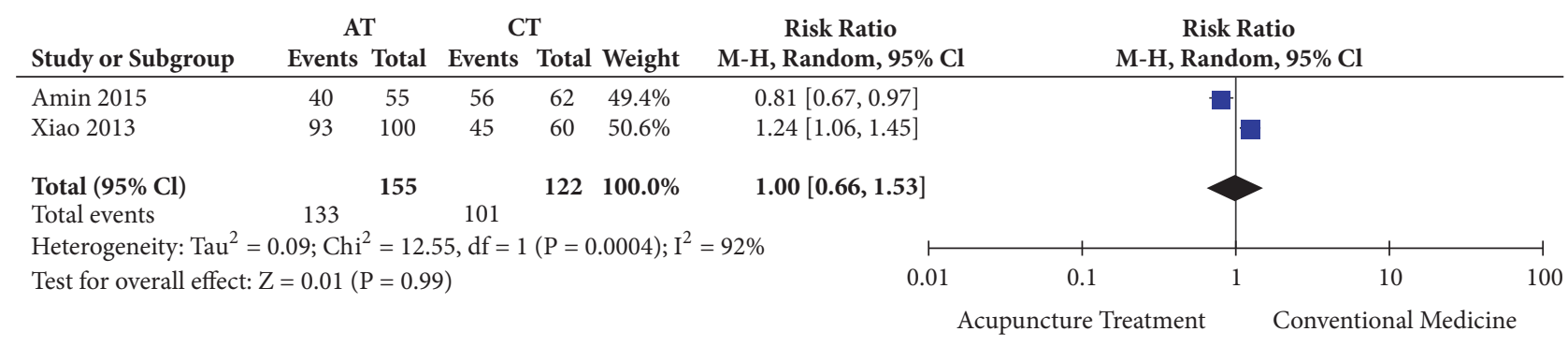

(a) Acupuncture treatment versus conventional treatment

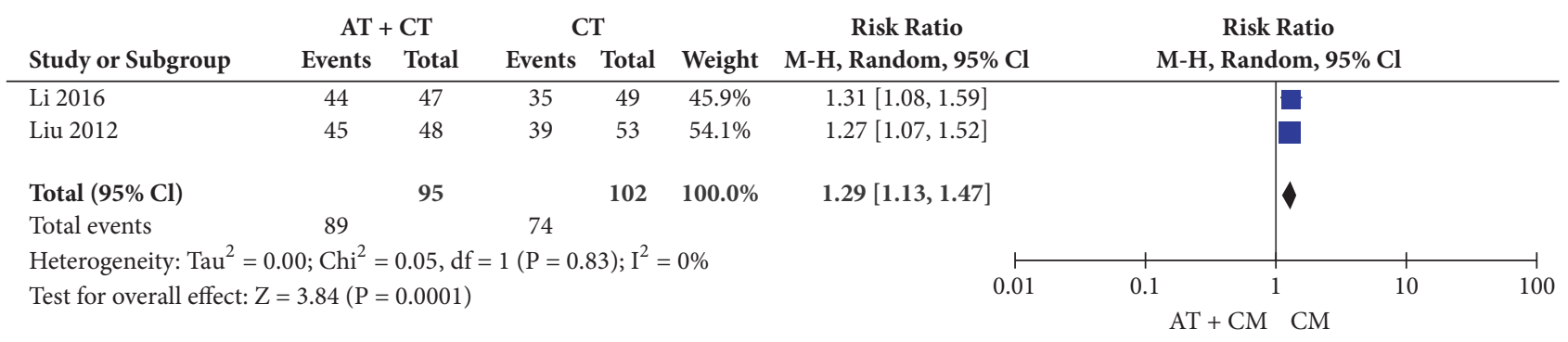

(b) Acupuncture treatment plus conventional treatment versus conventional treatment

FIGURE 2: Meta-analysis of total effectiveness rate for chronic pelvic pain. AT: acupuncture treatment; CT: conventional treatment; CI: confidence intervals.

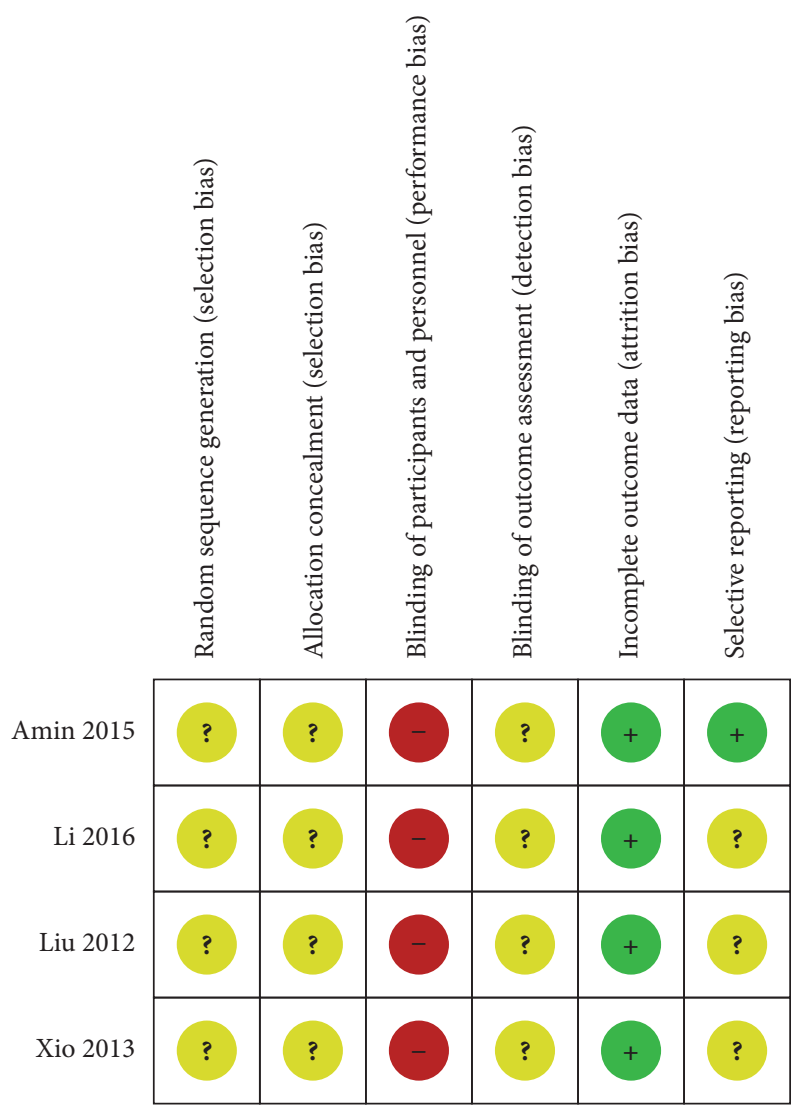

FIGURE 3: Summary risk of bias assessment.

differences in treatment type between groups. Moreover, these studies [8] did not report information about the blinding of the outcome assessors. All included RCTs [8] had a low risk of bias in addressing incomplete outcome data; three studies [8] had no missing outcome data; another trial [11] had missing outcome data, but the drop-out rate did not exceed $20 \%$ for short-term and $30 \%$ for long-term follow-up. In terms of selective reporting, only one [11] trial reported their protocol before conducting the studies.

\section{Discussion}

CPP in gynaecological practice is often associated with negative cognitive, behavioural, sexual, and emotional consequences and is often complex and difficult to treat [5]. Therapeutic options such as hormonal therapy or surgery are recommended in well-defined disease states and a multidisciplinary approach to pain management is used in persistent disease [5]. However in $30 \%$ of cases, no cause is ever determined and this presents a therapeutic challenge to the attending physician [5]. Thus, in the EAU guideline, the use of alternative therapies for chronic gynaecological pelvic pain is recommended [5].

Our systematic review provides suggestive evidence for the efficacy of AT, which is CAM therapy, in treating CPP. The meta-analysis that pooled data from two RCTs $[12,13]$ using the outcome measure of TER for CPP showed significant improvement with AT plus CT compared with CT alone (MD $=1.29, \mathrm{CI}=1.13$ to $\left.1.47, \mathrm{P}=0.0001, \mathrm{I}^{2}=0 \%\right)$. However, the meta-analysis in two studies $[11,14]$ indicated that AT showed no significant improvement on the outcome of TER for CPP compared to that with $\mathrm{CT}(\mathrm{MD}=1.00, \mathrm{CI}=0.66$ to 1.53 , $\left.\mathrm{P}=0.99, \mathrm{I}^{2}=92 \%\right)$. Although our findings indicated that AT can be recommended as additional treatment when CPP patients are treated with CT, there is insufficient evidence 
to recommend evidence-based treatment with AT for female CPP due to heterogeneity of control interventions and the small number of trials in the included studies.

The strength of our review is that we searched various databases without language restriction to avoid publication bias. Thus, three East Asian RCTs were included in the review; the researchers assess Chinese language articles.

Our review has several limitations. First, most of the included studies had low methodological quality in the Cochrane ROB assessment. None of the studies provided information on generation of random allocation and the method of allocation concealment. The blinding of participants, practitioners, and outcome assessors was not performed in all included RCTs. Low methodological quality RCTs led to overestimation of treatment effects [17]. Furthermore, three [12-14] of 4 studies did not provide a published protocol or register it prior to execution. Registration of clinical trial protocols is important to identify whether a trial is affected by selective or incomplete outcome reporting [18]. Future studies should be registered in an open-accessible registry such as ClinicalTrials.gov. or WHO.int/ICTRP [19, 20].

Second, in terms of safety, only one RCT [11] reported that AEs did not occur in 117 CPP patients. Safety is a fundamental principle in medical treatment. In general, there is a common impression that acupuncture is safer and is therefore recommended as alternative treatment. Recent research reported that minor and rare serious AEs can occur during acupuncture [21]. Therefore, AEs must be reported in RCTs of CPP in the future to draw firm conclusions on the severity and frequency of AEs due to AT.

Third, our findings were limited due to variation of AT types; three types of AT, including warm acupuncture, EA, and TET were included in the review. Traditionally, dry acupuncture needles have been used, and as new types of AT in combination with modern science and technology have been developed and utilized, the range of treatment tools is expanding. Thus, clinical studies according to different types of AT should be investigated in the future.

Lastly, the primary outcome of TER for CPP used in meta-analysis is not an internationally accepted tool for measuring pain. TER, an outcome measure generally used in China, was graded according to the following categories: clinical cure, markedly effective, effective, and ineffective [22]. The validity and reliability of TER have not yet been verified. In the future, internationally recognized measurements such as VAS or NRS should be used.

Although this review presented the applicability of AT for female CPP patients, standardization of AT intervention was not examined. Therefore, studies should consider the following factors: (1) AT type; (2) duration of treatment and number of treatment sessions based on each AT type; (3) size and depth of needle; (4) acupuncture points; and (5) appropriate placebo model for each AT type. Researchers need to investigate efficacy and safety of AT in CPP to establish CAM treatment guidelines that reflect our findings.

\section{Conclusion}

The results of our review and meta-analysis suggest the effectiveness of AT combined with CT for treating women with CPP compared to use of CT alone. However, current evidence is insufficient to verify the efficacy of AT for CPP because of the small number of RCTs and low methodological quality and heterogeneity of interventions. Therefore, larger, more rigorous and adequately powered multicentre RCTs are needed to provide clinical guidelines for AT in treating female CPP patients.

\section{Conflicts of Interest}

The authors declare that there are no conflicts of interest regarding the publication of this paper.

\section{References}

[1] ACOG Committee, "American College of Obstetricians and Gynecologists Practice Bulletin NO. 51. Chronic Pelvic Pain," Obstetrics \& Gynecology, vol. 103, no. 3, pp. 589-605, 2004.

[2] Y. C. Cheong, G. Smotra, and A. C. Williams, "Non-surgical Interventions for the Management of Chronic Pelvic Pain," Cochrane Library: Cochrane Reviews, vol. 3, no. Article ID CD008797, 2014.

[3] S. D. Mathias, M. Kuppermann, R. F. Liberman, R. C. Lipschutz, and J. F. Steege, "Chronic pelvic pain: Prevalence, healthrelated quality of life, and economic correlates," Obstetrics \& Gynecology, vol. 87, no. 3, pp. 321-327, 1996.

[4] Y. Cheong and R. William Stones, "Chronic pelvic pain: aetiology and therapy," Best Practice \& Research Clinical Obstetrics \& Gynaecology, vol. 20, no. 5, pp. 695-711, 2006.

[5] M. Fall, A. P. Baranowski, S. Elneil et al., "EAU guidelines on chronic pelvic pain," European Urology, vol. 57, no. 1, pp. 35-48, 2010.

[6] Korean Society of Obstetrics and Gynecology, Korean Traditional Medicine Obstetrics and Gynecology. 4th, Seoul, Euiseongdang Publishing Co, South Korea, 2016.

[7] J. Kim, N. Kang, M. Chae et al., "6 Case Series of the Chronic Pelvic Pain by Korean Medicine Therapies," The Journal of Oriental Obstetrics \& Gynecology, vol. 28, no. 2, pp. 143-155, 2015.

[8] Q. Yuan, P. Wang, L. Liu et al., "Acupuncture for musculoskeletal pain: A meta-analysis and meta-regression of sham-controlled randomized clinical trials," Scientific Reports, vol. 6, no. 1, 2016.

[9] L. Kalichman and S. Vulfsons, "Dry needling in the management of musculoskeletal pain," Journal of the American Board of Family Medicine, vol. 23, no. 5, pp. 640-646, 2010.

[10] Gihyun Lee and Woojin Kim, "The Modulatory Effect of Acupuncture on the Activity of Locus Coeruleus Neuronal Cells: A Review," Evidence-Based Complementary and Alternative Medicine, vol. 2017, Article ID 9785345, 8 pages, 2017.

[11] M. M. Amin, A. S. Ait-Allah, A. E.-S. A. Ali, R. A. Salem, S. R. Ahmed, and M. A. Alsammani, "Inferior hypogastric plexus blockade versus acupuncture for the management of idiopathic chronic pelvic pain: A randomized clinical trial," Biomedical Journal, vol. 38, no. 4, pp. 317-322, 2015.

[12] Z. S. Li, "Warm Acupuncture Therapy combine with Physical Therapy for Chronic Pelvic Pain caused by Pelvic Adhesion," 
Journal of Practical Traditional Chinese Medicine, vol. 32, no. 11, pp. 1047-1048, 2016.

[13] R. Liu, W. Su, and P. J. Sheng, "The Effect of the Warm Acupuncture and the Antibiotics Treatment for the Pelvic Cavity Pain of the Pelvic Inflammatory Disease," Medical Innovation of China, vol. 9, no. 32, pp. 13-14, 2012.

[14] H. Q. Xiao, "Therapeutic Observation on Acupoint Thread Embedding plus Auricular Point Sticking for Chronic Pelvic Pain after Acute Pelvic Infection," Shanghai J Acu-mox, vol. 32, no. 11, pp. 925-926, 2013.

[15] J. P. T. Higgins, D. G. Altman, P. C. Gøtzsche et al., "The Cochrane Collaboration's tool for assessing risk of bias in randomised trials," British Medical Journal, vol. 343, no. 7829, Article ID d5928, 2011.

[16] "Review Manager (RevMan) [Computer program], Version 5.3.5. Copenhage, The Nordic Cochrane Centre, The Cochrane Collaboration, 2014".

[17] K. F. Schulz, L. Chalmers, R. J. Hayes, and D. G. Altman, "Empirical evidence of bias: Dimensions of methodological quality associated with estimates of treatment effects in controlled trials," Journal of the American Medical Association, vol. 273, no. 5, pp. 408-412, 1995.

[18] Q. Zhang, J. Yue, B. Golianu, Z. Sun, and Y. Lu, "Updated systematic review and meta-analysis of acupuncture for chronic knee pain," Acupuncture in Medicine, vol. 35, no. 6, pp. 392-403, 2017.

[19] U. S. National Library, "U. S. National Library of Medicine," https://clinicaltrials.gov.

[20] World Health Organization, "International Clinical Trials Registry Platform," http://www.who.int/ictrp/en.

[21] M. W. Chan, X. Y. Wu, J. C. Wu, S. Y. Wong, and V. C. Chung, "Safety of Acupuncture: Overview of Systematic Reviews," Scientific Reports, vol. 7, no. 1, 2017.

[22] B. Yanju, L. Yang, B. Hua et al., "A systematic review and metaanalysis on the use of traditional Chinese medicine compound kushen injection for bone cancer pain," Supportive Care in Cancer, vol. 22, no. 3, pp. 825-836, 2014. 


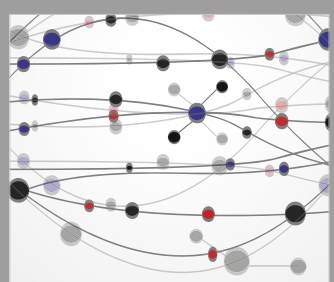

The Scientific World Journal
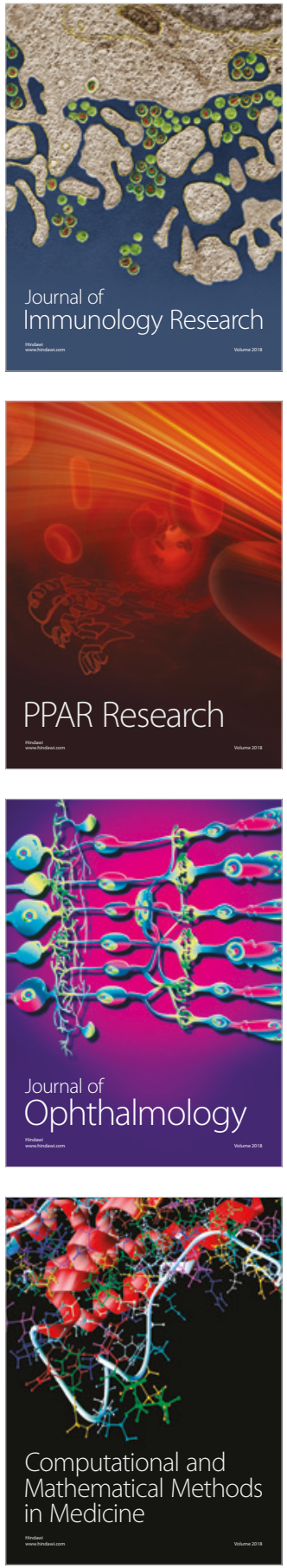

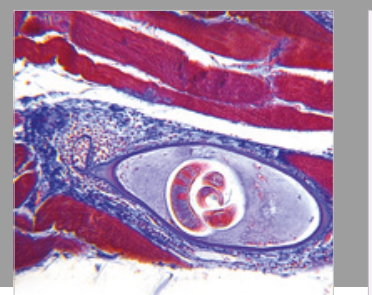

Gastroenterology Research and Practice

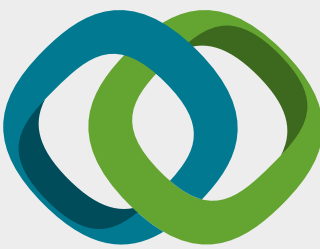

\section{Hindawi}

Submit your manuscripts at

www.hindawi.com
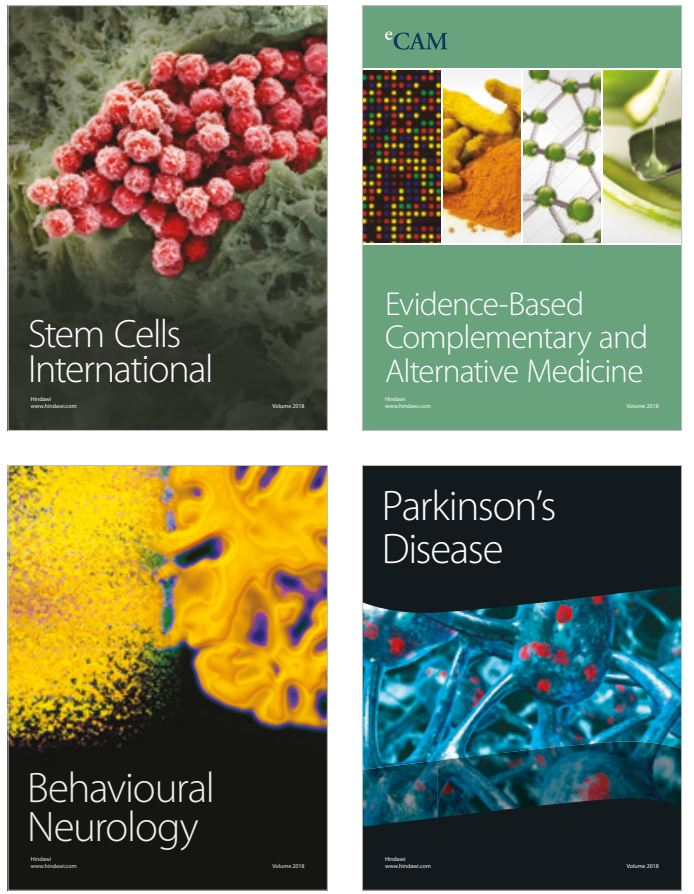

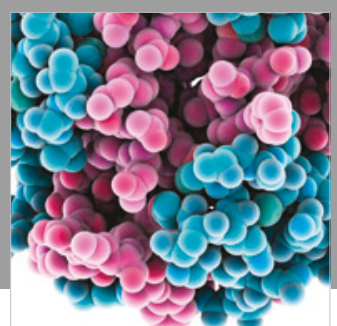

ournal of

Diabetes Research

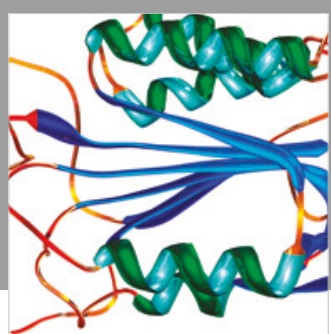

Disease Markers
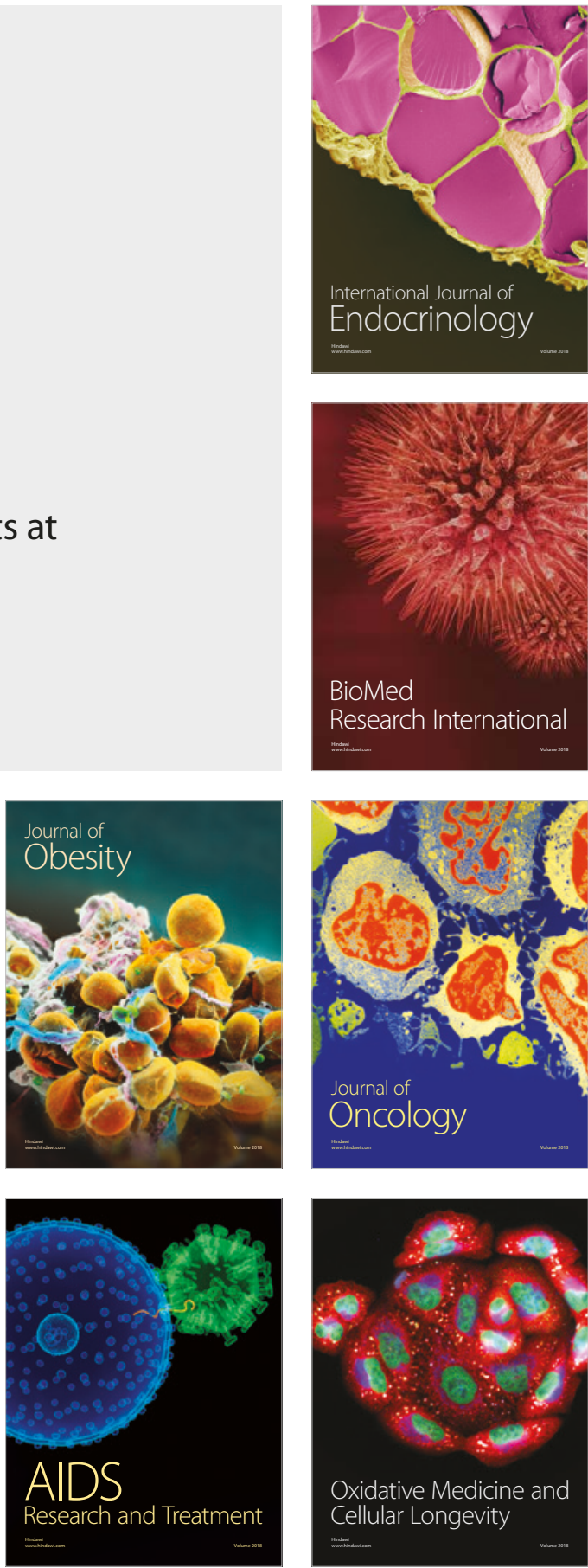